\title{
Excitations with negative dispersion in a spin vortex
}

\author{
M. Buess, ${ }^{1,2}$ T. P. J. Knowles, ${ }^{2}$ R. Höllinger, ${ }^{1}$ T. Haug, ${ }^{1}$ U. Krey,,${ }^{1}$ D. Weiss, ${ }^{1}$ D. Pescia, ${ }^{2}$ \\ M. R. Scheinfein, ${ }^{3}$ and C. H. Back ${ }^{1}$ \\ ${ }^{1}$ Institut für Experimentelle und Angewandte Physik, Universität Regensburg, Universitätsstrasse 31, 93040 Regensburg, Germany \\ ${ }^{2}$ Laboratorium für Festkörperphysik, Eidgenössische Technische Hochschule Zürich, CH-8093 Zürich, Switzerland \\ ${ }^{3}$ Simon Fraser University, 8888 University Drive, Burnaby, BC, Canada U5A 156 \\ (Received 21 September 2004; revised manuscript received 9 November 2004; published 23 March 2005)
}

\begin{abstract}
Micron-sized ferromagnetic permalloy disks having an in-plane vortexlike configuration are excited by a fast-rise-time magnetic-field pulse perpendicular to the plane. The excited modes are imaged using timeresolved magneto-optic Kerr microscopy and Fourier transformation. Two types of modes are observed: modes with circular nodes and modes with diametric nodes. The frequency of the modes with circular nodes increases with the number of nodes. In contrast, the frequency of the modes with diametric nodes decreases with the number of nodes. This behavior is explained accurately by an analytical model.
\end{abstract}

DOI: 10.1103/PhysRevB.71.104415

PACS number(s): 75.40.Gb, 75.75.+a, 75.60.-d

Acoustic membranes resonate in such a way that adding a circular or a diametric node inevitably produces an increase of the vibrational frequency (Chladni's law $\left.{ }^{1,2}\right)$. This phenomenon is a consequence of the positive dispersion of ordinary waves. Negative dispersion-the lowering of the frequency with increasing wave vector-is occasionally measured in light optics for negative index media, ${ }^{3,4}$ special photonics crystals, ${ }^{5,6}$ and media with inverted population. ${ }^{7}$ In spin systems, negative group velocity was predicted theoretically ${ }^{8-10}$ and excitations with frequencies lower than the uniform precessional mode were interpreted as magnetostatic backward modes. ${ }^{11-16}$ We demonstrate by directly imaging the nodal structure that Chladni's law is violated by spin excitations in small circular ferromagnetic disks with a vortexlike groundstate configuration. While the precessional frequency indeed increases upon adding circular nodes, it decreases when diametric nodes are built in. We argue that this violation is due to the particular laws governing spin dynamics. This phenomenon can be interpreted as the spin analogon of a photonic left-handed material.

Here we study the excitation spectrum of individual micron-sized permalloy disks with a vortex structure excited by a perpendicular magnetic-field pulse. The magnetic response of the individual disks is examined by recording a time-resolved movie with a high spatial resolution of $300 \mathrm{~nm}$. In the Fourier transform of the movies, we can identify the eigenmodes up to the third order in this highly symmetric system. The excitation spectrum and spatial distribution of all modes was imaged for a circular platelet with a radius of $3 \mu \mathrm{m}$ and a thickness of $15 \mathrm{~nm}$ in a recent paper ${ }^{25}$ in more detail. Here we present further experimental results by including additional samples. The measured eigenmodes can be accounted for by a linear model based purely on dipolar interactions. We expand our analytical model ${ }^{17}$ to include modes containing diametric nodal lines in order to construct a more thorough theoretical model. Micromagnetic simulations using the Landau-Lifshitz-Gilbert (LLG) $\operatorname{code}^{18}$ and the object oriented micromagnetic framework 19 (OOMMF) support our experimental findings and the analytical results. In the size regime we examined, no deviations of the modal frequencies from the dipolar-dominated model are observed. ${ }^{33}$

The samples studied are single ferromagnetic permalloy disks with a thickness $d=15 \mathrm{~nm}$, ranging in radius from 1 to $3 \mu \mathrm{m}$ and patterned by e-beam lithography onto a Si substrate. A 500-nm-thick $\mathrm{Cu}$ microcoil was prepared around the element using e-beam lithography and electroplating. It surrounds the sample with an inner diameter of $8 \mu \mathrm{m}$ and an outer diameter of $12 \mu \mathrm{m}$. For details of the preparation see Ref. 20.

In the ground state the magnetic elements exhibit a flux closure vortex configuration. ${ }^{20} \mathrm{We}$ perturb this state by launching a short current pulse though the microcoil that produces a magnetic tipping field pulse perpendicular to the sample plane. The maximum strength of the field is less than 50 Oe, with a rise time of about 100 ps. This magnetic-field pulse exerts a torque onto the local magnetization vector that launches the precessional motion of the elements magnetization. The motion is imaged stroboscopically by means of the magneto-optic Kerr effect as a function of the time delay between application of the tipping pulse and the probe pulse. Details of the experiment are described elsewhere. ${ }^{17,21-25}$ For each magnetic element a movie of the $z$ component of the magnetization vector is recorded at time intervals of $25-40 \mathrm{ps}^{25}$ From such a sequence one may extract an overall periodicity corresponding to the strongest excited mode. The spin motion, however, is not uniform, but is the superposition of a number of modes. ${ }^{13,14,25}$

Using a recently developed phase-sensitive Fourier transform procedure, ${ }^{13,14,25}$ we are able to reconstruct each individual mode driving the spin motion. Let us briefly describe the method: the Fourier transform of the time-domain data is performed at each location on the disk. Both the amplitude and the phase are recorded. The Fourier transform consists of a sequence of resonances marking the characteristic eigenmodes. The frequencies corresponding to the maxima of the resonance peaks are used for constructing the experimental dispersion curves. The amplitude (left-hand side) and the phase (right-hand side) at resonance are plotted in Fig. 1. One recognizes two types of modes. Some are organized into concentric rings with circular nodes [Figs. 1(a)-1(c)]. The 


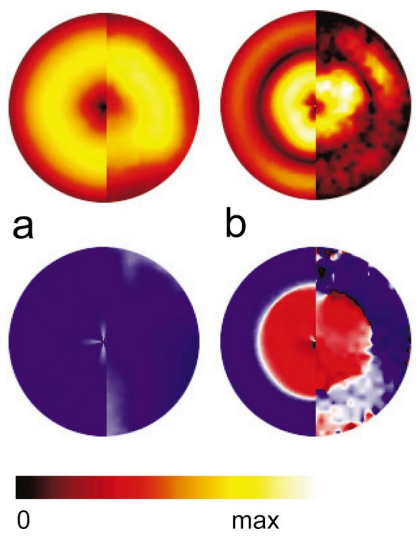

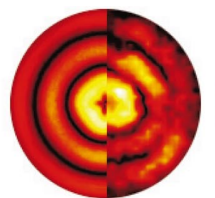

C

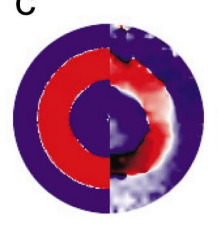

d
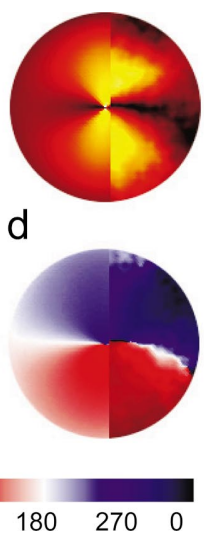

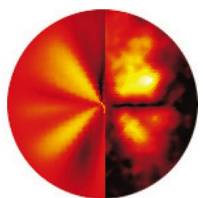

e

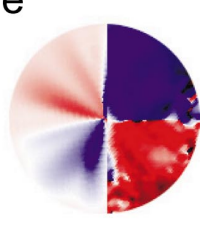

FIG. 1. (Color) Images of the eigenmodes for $R=3 \mu \mathrm{m}$. The top row shows the absolute value of the Fourier amplitude at resonance, the bottom row the phase. The modal maps are composed from two half-images: the left from the micromagnetic simulation; the right from the experiment. (a)-(c): Axially symmetric modes showing concentric nodes $(n=1,2,3, m=0)$. (a) $2.80 \mathrm{GHz}$. (b) $3.91 \mathrm{GHz}$. (c) $4.49 \mathrm{GHz}$. (d), (e): These modes have nodal lines going across the image. (d) $2.08 \mathrm{GHz}$, one azimuthal node (1,1). (e) 1.69 GHz, two azimuthal nodes (1,2). Notice that across the nodal lines the phase changes by $180^{\circ}$. A Hamming cutoff window was used to obtain the fast Fourier transform (FFT) data from the 3.33-ns-long scans. The Fourier spectrum consists of a sequence of resonances along the frequency axis corresponding to the eigenmodes. Although the spacing in the frequency domain of the raw data is $d f=1 /\left(T_{\text {end }}-T_{\text {start }}\right)=1 / 3.33 \mathrm{~ns}=0.30 \mathrm{GHz}$, the exact location of the maximum of these resonances can be determined with a higher accuracy when using zero filling. The half-images from the timedomain simulation data were obtained using the same method.

modes in Figs. 1(d) and 1(e) instead have one (respectively two) diametric nodes. The various nodes are easily identified because at their spatial location the spectral weight is small and because the phase jumps by $\pi$. The occurrence of these modes - in particular of those with diametric nodes-is discussed in detail in Ref. 25.

We relate the nodal structure of the modes to a $\vec{k}$ vector and construct experimental dispersion relations. The modal structure observed in Fig. 1 requires the introduction of polar coordinates $r, \varphi$ in the plane of the disk. We define the wave vector $\vec{k}=\left(k_{r}, k_{\varphi}\right)$ of a spin excitation by introducing an orthogonal set of basis functions suitable for the two-dimensional vortexlike spin configuration $m_{r}(r, \varphi) \alpha J_{1}\left(k_{r} r\right) \exp \left(i k_{\varphi} \varphi\right)$. In Appendix A we show that the radial part is an exact solution for infinite radius. ${ }^{17}$ These functions are the analog for circular geometries of plane waves in Cartesian geometries. The $2 \pi$ periodicity in $\varphi$ requires $k_{\varphi}^{m}=0, \pm 1, \pm 2, \ldots$, with the index $m=0, \pm 1, \pm 2, \ldots$ counting the number of diametric nodes. The boundary conditions at $r=0$ and at $r=R$ [Refs. (16 and 17)] establish a set of possible values for $k_{r}: k_{r}^{n}=x_{n} / R, x_{n}$ indicating the zeroes of the Bessel functions $J_{1} ; n \in \mathcal{N}$ counts the number of circular antinodes. $n=1$ corresponds to the state with nodes at $r=R$ and $r=0$. From Fig. 1 -the nodes and their location-one can read out the mode numbers $(n, m)$ of the measured modes. We can now construct the experimental dispersion relations $f$ vs $(n, m=0)$ [radial modes, Fig. 2(a)] and $f$ vs $(n=1, m)$ [azimuthal modes, Fig. 2(b)]. We observe a positive dispersion for the axially symmetric modes $m=0$ and negative dispersion for the modes with $m \neq 0$. In contrast, Chladni's law for sound in a circular membrane ${ }^{1,2}$ states that $f \propto(m+2 n)^{2}$. Figure 2 reports the experimental data for disks with different diameters. The dispersion curves show the same behavior for all disks, their sign being independent of the diameter.
In the following, we suggest a quantitative model that essentially accounts for all the experimental dispersion curves measured, including the lowering of $f$ with $m$ and the size dependence. We point out that for moderate field pulse excitations and large magnetic elements (e.g., several tens of microns) the highly degenerate excitation spectrum is dominated by magnetostatic modes. ${ }^{23}$ When the size of the elements is reduced or higher order modes are excited, the exchange interactions can, in general, no longer be ignored and the dynamic response gradually changes from a purely magnetostatic to an exchange-dominated one. ${ }^{15,26,27}$ Yet, on the basis of the recent findings by Park and co-workers ${ }^{26}$ and on
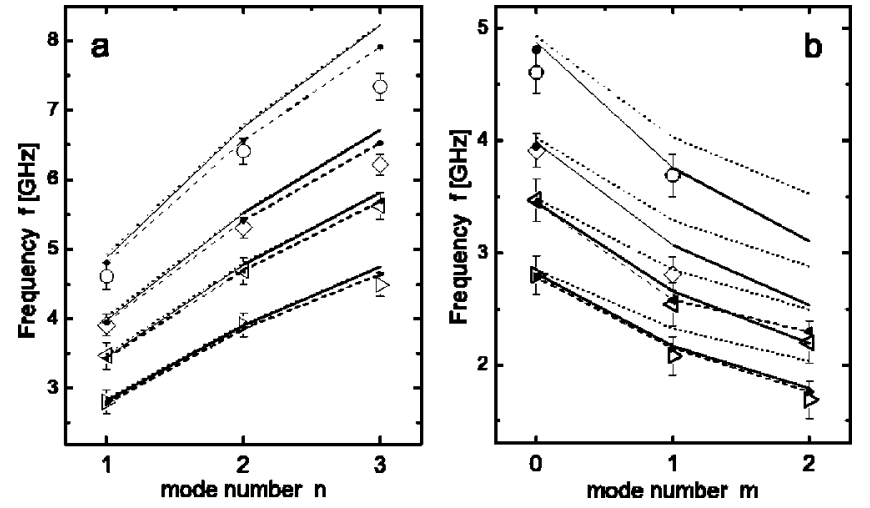

FIG. 2. Dispersion relations. (a) The frequency for modes of the type $(n, m=0)$ as a function of the radial mode number $n: R$ $=1 \mu \mathrm{m}(\bigcirc), 1.5 \mu \mathrm{m}(\diamond), 2 \mu \mathrm{m}(\triangle), 3 \mu \mathrm{m}(\nabla)$. The error bars are $\pm d f / 2$. (b) The frequency for modes of the type $(n=1, m)$ as a function of the azimuthal mode number $m$. The corresponding data points of the micromagnetic simulation based on the LLG code $^{18}$ are diagramed as small full symbols and dashed lines. Results from the variational model as discussed in the text are represented as solid and the dotted lines. The dotted line is calculated by the diagonal elements and the solid line from the full matrix eigenvalues. 
the results we are going to present, we believe that the excitation spectrum in mesoscopic permalloy elements with the diameters considered here can be accounted for by considering only the dipolar interaction. Thus, as shown previously, ${ }^{17}$ the relevant operator governing the dipolar modes is the dimensionless in-plane radial field given by

$$
\hat{h}_{r}\left[m_{r}\right]=d \frac{\partial}{\partial r} \int_{\text {disk }} d^{2} \vec{r}^{\prime} \frac{1}{\left|\vec{r}-\vec{r}^{\prime}\right|} \frac{1}{r^{\prime}} \frac{\partial}{\partial r^{\prime}}\left[r^{\prime} m_{r}\left(r^{\prime}, \varphi^{\prime}\right)\right] .
$$

The eigenfrequencies of the various modes are related to the eigenvalues $N_{r}$ of the equation $\hat{h}_{r}\left[m_{r}\right]=-N_{r} m_{r}$ by the relation ${ }^{17} \omega^{2}=4 \pi\left(\gamma M_{s}\right)^{2} N_{r}$. These results were already published in Ref. 17 and used there to calculate the modes with circular nodes. Here we generalized them to include modes with diametric nodes as well. Generalizing Ref. 17, the matrix $[h]_{n n^{\prime}, m} \doteq\left(\psi_{n, m}, \hat{h}_{r} \psi_{n^{\prime}, m}\right)$ within the trial space consisting of orthonormal basis functions ${ }^{28}$

$$
\psi_{n, m} \doteq \frac{1}{\sqrt{\pi} R J_{2}\left(x_{1 n}\right)} J_{1}\left(k_{n} r\right) e^{i m \varphi}
$$

is diagonalized. Using 15 basis functions $n, n^{\prime}=1, \ldots, 15$ within each sector $m$ was found to give good convergence. The details of this calculation are provided in Appendix B. The $m=0$ sector provides the eigenvalues for the modes with circular nodes. The $m=1,2, \ldots$ provides the eigenvalues for modes with $1,2, \ldots$ diametric nodes. The frequencies resulting from the matrix diagonalization are plotted as dotted lines in Fig. 2. The agreement with the experimental data is remarkable, taking into account that the calculation is a fully analytical one with no adjustable parameters. In particular, the negative dispersion for the modes with diametric nodes is also well reproduced.

The central result of this paper is the existence of two distinct types of dynamical modes, one with positive dispersion and one with negative dispersion. The references ${ }^{8-16}$ quoted here (the list is by no means exhaustive) indicate that this should be a quite general phenomenon, not limited to the geometry and size investigated in the present paper, when the excitation spectrum is dominated by magnetostatic modes. As suggested by Fletcher and Kittel more than 40 years ago (read the last eight lines of Ref. 9), a possible way to qualitatively explain this phenomenon is to consider the magnetostatic energy associated with the excited modes. In Appendix B we show that the magnetostatic energy Fletcher and Kittel are referring to are the diagonal elements of our matrix. These diagonal elements are exactly the Coulomb energy of the effective magnetic charge $(1 / r)(\partial / \partial r) r \psi_{n, m}(r, \varphi)$. They are also indicated in Fig. 2 and are clearly quite close to the "true" eigenfrequencies, confirming the qualitative suggestion by Fletcher and Kittel. The negative dispersion means that when the magnetic charge distribution is partitioned by introducing diametric nodes, the Coulomb energy (and thus the frequency) decreases. In light of these considerations, the origin of the violation of Chladni's law in this spin system is evident: in ordinary standing waves residing on a membrane, the change of frequency due to diametric modes is related to the matrix elements of the angular part of

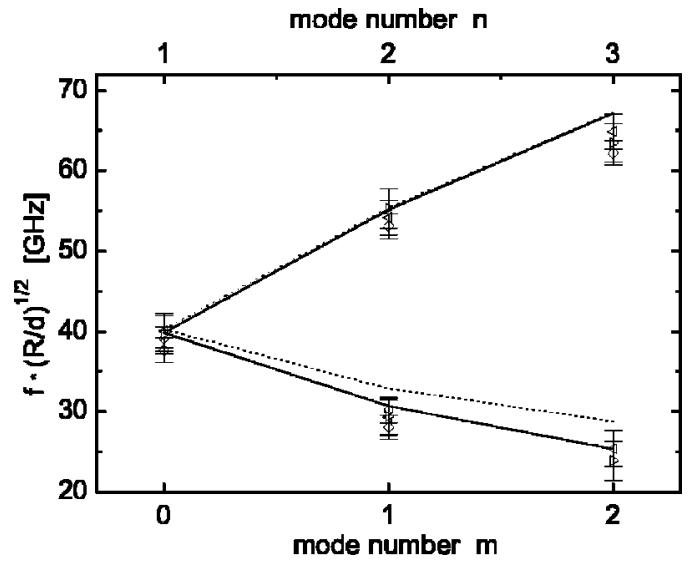

FIG. 3. Scaling. When $f(n, m)(R / d)^{1 / 2} \doteq \tilde{f}$ is plotted vs $m$ at fixed $n$ or vs $n$ at fixed $m$, all data points for different radii fall onto one single dispersion curve $\widetilde{f}(m)$ and $\widetilde{f}(n)$. The symbol and line attributes are the same as in Fig. 2.

the Laplace operator, which scales with the square of the angular momentum, $m^{2}$. The relevant operator for spin systems contains the Coulomb interaction, which behaves quite differently with $m$, as discussed above.

Our analytical calculations (see Appendix B) suggest a simple scaling law that should be obeyed by all modes, provided they are of magnetostatic origin: all frequencies for different radii should fall onto one single function when $f(n, m)(R / d)^{1 / 2}$ is plotted as a function of $n$ at fixed $m$ (radial modes) or $m$ at fixed $n$ (azimuthal modes), this function being only dependent on the number of diametric and circular nodes. The agreement is approximately realized (see Fig. 3). The largest deviations from this scaling law are observed for small radii, where the exchange interaction is expected to become more important. We recall that the frequency depends only on the symmetry function $f(n, m)$ and the aspect ratio of the disk $(f \propto \sqrt{d / R})$ for both kind of modes. This is quite an universal behavior for these idealized magnetostatic thin film vortex structures.

Based on these considerations, a negative dispersion in magnonic crystals seems to be a quite common phenomenon. The argument developed above shows that introducing spatial nodes does not necessarily mean that the frequency increases, as expected for a large class of phenomena such as ordinary waves but also for quantum-mechanical systems like the hydrogen atom or the harmonic oscillator. In smaller magnetic disks, the exchange interaction should become more important, and it is expected to change the sign of the dispersion. ${ }^{9}$ We can envisage the realization of a type of junction between elements having opposite dispersion, in analogy to the boundary between left- and right-handed photonic crystals. ${ }^{3,4}$ If the analogy holds true, a spin-wave excitation with $m \neq 0$ - such as those made visible in Ref. 25should change their helicity when sent across such a junction, ${ }^{29}$ thus establishing an elementary spin-logic device with subnanosecond switching time.

D. P. would like to thank V. Pokrovsky and G.M. Graf for fruitful discussions. Financial support by the Schweizerische Nationalfonds, ETH Zurich and the Deutsche Forschungsge- 
meinschaft through the Forschergruppe FOG370/2-1 and the priority program SPP1133 is gratefully acknowledged.

\section{APPENDIX A}

Here we show that the trial function $m_{r}=J_{1}\left(k_{r} r\right)$ used above is an exact solution for the infinite radius limit of the eigenvalue problem. We substitute $m_{r}=J_{1}\left(k_{r} r\right)$ $=-\left(1 / k_{r}\right)(\partial / \partial r) J_{0}\left(k_{r} r\right)$ in Eq. (1) and obtain

$$
\hat{h}_{r}\left[m_{r}\right]=-\frac{d}{k_{r}} \frac{\partial}{\partial r} \int_{0}^{\infty} d^{2} \vec{r}^{\prime} \frac{1}{\left|\vec{r}-\vec{r}^{\prime}\right|} \frac{1}{r^{\prime}} \frac{\partial}{\partial r^{\prime}}\left(r^{\prime} \frac{\partial}{\partial r^{\prime}} J_{0}\left(k_{r} r^{\prime}\right)\right) .
$$

Expanding $\quad 1 /\left|\vec{r}-\vec{r}^{\prime}\right|=\sum_{l=-\infty}^{\infty} \int_{0}^{\infty} d k J_{l}(k r) J_{l}\left(k r^{\prime}\right) e^{i l\left(\varphi-\varphi^{\prime}\right)} \quad$ into Bessel functions and using the Bessel differential equation $r \partial_{r}\left[r \partial_{r} J_{0}\left(k_{r} r\right)\right]+k_{r}^{2} r^{2} J_{0}\left(k_{r} r\right)=0$, we obtain

$$
\begin{aligned}
\hat{h}_{r}\left[m_{r}\right]= & k_{r} d \frac{\partial}{\partial r} \int_{0}^{\infty} r^{\prime} d r^{\prime} \int_{0}^{2 \pi} d \varphi^{\prime} \int_{0}^{\infty} d k \sum_{l=-\infty}^{\infty} e^{i l\left(\varphi-\varphi^{\prime}\right)} \\
& \times J_{l}(k r) J_{l}\left(k r^{\prime}\right) J_{0}\left(k_{r} r^{\prime}\right) .
\end{aligned}
$$

Because $\int_{0}^{2 \pi} d \varphi^{\prime} e^{i l \varphi^{\prime}}=2 \pi$ only for $l=0$, only the $l=0$ summand contributes

$$
\hat{h}_{r}\left[m_{r}\right]=-2 \pi k_{r} d \int_{0}^{\infty} r^{\prime} d r^{\prime} \int_{0}^{\infty} d k J_{0}(k r) J_{0}\left(k r^{\prime}\right) J_{0}\left(k_{r} r^{\prime}\right) .
$$

The integral relation ${ }^{30} \quad\left(1 / k_{r}\right) \delta\left(k-k_{r}\right)$ $=\int_{0}^{\infty} r^{\prime} d r^{\prime} J_{l}\left(k r^{\prime}\right) J_{l}\left(k_{r} r^{\prime}\right)$ is used to obtain

$$
\begin{aligned}
-2 \pi k_{r} d \frac{\partial}{\partial r} \int_{0}^{\infty} d k \frac{\delta\left(k-k_{r}\right)}{k_{r}} J_{0}(k r)= & \\
& -2 \pi d \frac{\partial}{\partial r} J_{0}\left(k_{r} r\right) \stackrel{!}{=}-\frac{N_{r}}{k_{r}} \frac{\partial}{\partial r} J_{0}\left(k_{r} r\right),
\end{aligned}
$$

i.e., the dispersion relation

$$
N_{r}=2 \pi d k_{r} .
$$

\section{APPENDIX B}

We want to derive a model for spin excitations of magnetostatic origin. We consider thin micron-sized disks with a ground-state circulating flux-closure configuration $\left(M_{r}=0\right.$, $\left.M_{\varphi}=M_{s}, M_{z}=0\right)$, subject to the linearized Landau-Lifshitz equation

$$
\begin{gathered}
\dot{m}_{r}=\gamma m_{z} 4 \pi M_{s}, \\
\dot{m}_{\varphi}=0, \\
\dot{m}_{z}=-\gamma \hat{h}_{r}\left[m_{r}\right] M_{s} .
\end{gathered}
$$

Here, $M_{s}$ is the saturation magnetization, and $\gamma$ is the absolute value of the gyromagnetic ratio. The equations can be decoupled yielding oscillating solutions $m_{r}, m_{z} \sim \exp (i \omega t)$ with the frequency $\omega^{2}=4 \pi\left(\gamma M_{s}\right)^{2} N_{r}$. These solutions belong to the eigenvalue equation ${ }^{17}$

$$
\hat{h}_{r}\left[m_{r}\right]=-N_{r} m_{r}
$$

$\hat{h}_{r}$ is the dimensionless operator for the $r$ component of the magnetization provided by the dipolar field of magnetostatic origin and can be written as a Coulomb-type interaction for the magnetic-charge distribution $(1 / r)(\partial / \partial r)\left[r m_{r}(r, \varphi)\right]$

$$
\hat{h}_{r}\left[m_{r}\right]=d \frac{\partial}{\partial r} \int_{\text {disk }} d^{2} r^{\prime} \frac{1}{\left|\vec{r}-\vec{r}^{\prime}\right|} \frac{1}{r^{\prime}} \frac{\partial}{\partial r^{\prime}}\left[r^{\prime} m_{r}\left(r^{\prime}, \varphi^{\prime}\right)\right] .
$$

This is valid as long as the spin motion is dominated by the dipolar interaction. ${ }^{16,17,23}$ In the range of radii explored in this study this assumption seems to be justified, i.e., the exchange interaction plays a negligible role. This is due to the fact that the spatial scale of the excitations observed is much larger than the exchange length. ${ }^{17}$

We now need to solve the eigenvalue problem Eq. (B2). Here we propose to use a variational approach: we calculate the matrix elements of the operator (B3) in the basis

$$
\mathbb{B}=\left\{\psi_{n, m}\right\}_{n=1,2,3, \ldots, m=0, \pm 1, \pm 2, \ldots},
$$

where

$$
\psi_{n, m}=\frac{1}{\sqrt{\pi} R J_{2}\left(x_{1 n}\right)} J_{1}\left(k_{r}^{n} r\right) e^{i m \varphi},
$$

and $k_{r}^{n}=x_{1 n / R}$ and $x_{1 j}$ are the zeros $x_{11}=3.83, x_{12}=7.02, x_{13}$ $=10.17, \ldots$ of the Bessel function $J_{1}(x) . n=1,2,3, \ldots$ is the circular (or radial) mode number and $m=0, \pm 1, \pm 2, \ldots$ is the diametric (azimuthal) mode number. The set $\mathbb{B}$ is an orthonormal basis on the circle of radius $R$ with respect to the scalar product $^{28}$

$$
\begin{aligned}
\left\langle\psi_{n, m}, \psi_{n^{\prime}, m^{\prime}}\right\rangle & =\int_{0}^{R} \int_{0}^{2 \pi} r d r d \varphi \psi_{n, m}^{*}(r, \varphi) \psi_{n^{\prime}, m^{\prime}}(r, \varphi) \\
& =\delta_{n n^{\prime}} \delta_{m m^{\prime}} .
\end{aligned}
$$

Our motivation for this choice of basis is twofold. First, in the context of the variational method, the convergence of the eigenvalues with increasing trial space size is faster if the trial functions are close to the real eigenfunctions. In the present case, experiment suggests that the choice of $J_{1}$ is plausible (see Fig. 1). Second, we know that the functions $J_{1}\left(k_{r}^{n} r\right)$ are exact solutions for the infinite radius eigenvalue problem (see Appendix A).

The matrix element $[h]_{n n^{\prime}, m}$ corresponding to the states with angular momentum $m$ reads 


$$
\begin{aligned}
{[h]_{n n^{\prime}, m}=} & \left\langle\psi_{n, m}, h_{r} \psi_{n^{\prime}, m}\right\rangle \\
= & \int_{\text {disk }} d^{2} r \psi_{n, m}^{*}(r, \varphi) d \frac{\partial}{\partial r} \\
& \times\left\{\int_{\text {disk }} d^{2} r^{\prime} \frac{1}{\left|\vec{r}-\vec{r}^{\prime}\right|} \frac{1}{r^{\prime}} \frac{\partial}{\partial r^{\prime}}\left[r^{\prime} \psi_{n^{\prime}, m}\left(r^{\prime}, \varphi\right)\right]\right\}
\end{aligned}
$$

Partial integration yields a symmetric form in $r$ and $r^{\prime}$, which can be interpreted as a magnetic Coulomb energy of the magnetic-charge distributions $(1 / r)(\partial / \partial r)[r \psi(r, \varphi)]$

$$
\begin{aligned}
{[h]_{n n^{\prime}, m}=} & -d \int_{\text {disk }} \int_{\text {disk }} d^{2} r d^{2} r^{\prime}\left\{\frac{1}{r} \frac{\partial}{\partial r}\left[r \psi_{n, m}^{*}(r, \varphi)\right]\right\} \\
& \times \frac{1}{\left|\vec{r}-\vec{r}^{\prime}\right|}\left\{\frac{1}{r^{\prime}} \frac{\partial}{\partial r^{\prime}}\left[r^{\prime} \psi_{n^{\prime}, m}\left(r^{\prime}, \varphi\right)\right]\right\}
\end{aligned}
$$

since the boundary terms

$$
\begin{aligned}
& d\left\{\int_{0}^{2 \pi} d \varphi r \psi_{n, m}^{*}(r, \varphi) \int_{\text {disk }} d^{2} r^{\prime} \frac{1}{\left|\vec{r}-\vec{r}^{\prime}\right|} \frac{1}{r^{\prime}} \frac{\partial}{\partial r^{\prime}}\right. \\
& \left.\quad \times\left[r^{\prime} \psi_{n^{\prime}, m}\left(r^{\prime}, \varphi^{\prime}\right)\right]\right\}_{R}^{0}
\end{aligned}
$$

vanish under the boundary condition $\psi_{n, m}^{*}(r=0, \varphi)$ $=\psi_{n, m}^{*}(r=R, \varphi)=0$. Using the Bessel recursion relation $\left.(1 / r)(\partial / \partial r) r J_{1}\left(k_{r}^{n} r\right)\right]=\kappa J_{0}\left(k_{r}^{n}\right)$ gives

$$
\begin{aligned}
{[h]_{n n^{\prime}, m}=} & -\frac{1}{\pi R^{2} J_{2}\left(x_{1 n}\right) J_{2}\left(x_{1 n^{\prime}}\right)} \frac{x_{1 n}}{R} \frac{x_{1 n^{\prime}}}{R} \\
& \times d \int_{\text {disk }} \int_{\text {disk }} d^{2} r d^{2} r^{\prime} J_{0}\left(k_{r}^{n} r\right) J_{0}\left(k_{r}^{n^{\prime}} r^{\prime}\right) \\
& \times e^{-i m\left(\varphi^{\prime}-\varphi\right)} \frac{1}{\left|\vec{r}-\vec{r}^{\prime}\right|} .
\end{aligned}
$$

Expanding in Bessel functions gives

$$
\begin{aligned}
{[h]_{n n^{\prime}, m}=} & -\frac{1}{\pi R^{2} J_{2}\left(x_{1 n}\right) J_{2}\left(x_{1, n^{\prime}}\right)} \frac{x_{1 n}}{R} \frac{x_{1 n^{\prime}}}{R} \\
& \times d \int_{0}^{R} r d r \int_{0}^{2 \pi} d \varphi \int_{0}^{R} r^{\prime} d r^{\prime} \int_{0}^{2 \pi} d \varphi^{\prime} J_{0}\left(k_{r}^{n} r\right) \\
& \times J_{0}\left(k_{r}^{n^{\prime}} r^{\prime}\right) e^{i m\left(\varphi^{\prime}-\varphi\right)} \sum_{l=-\infty}^{\infty} \int_{0}^{\infty} d k J_{l}(k r) J_{l}\left(k r^{\prime}\right) e^{i l\left(\varphi-\varphi^{\prime}\right)} .
\end{aligned}
$$

The Fourier orthogonality relations

$$
\int_{0}^{2 \pi} d \varphi \int_{0}^{2 \pi} d \varphi^{\prime} e^{-i m\left(\varphi-\varphi^{\prime}\right)} e^{i l\left(\varphi-\varphi^{\prime}\right)}=(2 \pi)^{2} \delta_{l m}
$$

give

$$
\begin{aligned}
{[h]_{n n^{\prime}, m}=} & -\frac{(2 \pi)^{2}}{\pi R^{2} J_{2}\left(x_{1 n}\right) J_{2}\left(x_{1 n^{\prime}}\right)} \frac{x_{1 n}}{R} \frac{x_{1 n^{\prime}}}{R} \\
& \times d \int_{0}^{R} r d r \int_{0}^{R} r^{\prime} d r^{\prime} J_{0}\left(k_{r}^{n} r\right) J_{0}\left(k_{r}^{n^{\prime}} r^{\prime}\right) \\
& \times \int_{0}^{\infty} d k J_{m}(k r) J_{m}\left(k r^{\prime}\right) .
\end{aligned}
$$

Introducing the dimensionless variables $x=r / R, y=r^{\prime} / R$ and $\tilde{k}=k R$ leads to

$$
\begin{aligned}
{[h]_{n n^{\prime}, m}=} & -\frac{d(2 \pi)^{2} x_{1 n^{1 n^{\prime}}} x_{1 n^{\prime}}}{\pi J_{2}\left(x_{1 n}\right) J_{2}\left(x_{1 n^{\prime}}\right)} \\
& \times \int_{0}^{1} x d x \int_{0}^{1} y d y J_{0}\left(x_{1 n^{n}} x\right) J_{0}\left(x_{1 n^{\prime}} y\right) \\
& \times \int_{0}^{\infty} \frac{d \tilde{k}}{R} J_{m}(\tilde{k} x) J_{m}(\tilde{k} y)
\end{aligned}
$$

The last integral has a closed form result in hypergeometric functions $^{31}$

$$
\begin{aligned}
\int_{0}^{\infty} d \tilde{k} J_{m}(\tilde{k} x) J_{m}(\tilde{k} y)= & \frac{(2 m) !}{(m !)^{2} 2^{2 m}} \frac{(x y)^{m}}{\left(x^{2}+y^{2}\right)^{m+1 / 2} 2} F_{1} \\
& \times\left(\frac{2 m+1}{4}, \frac{2 m+3}{4}, m+1, \frac{4 x^{2} y^{2}}{\left(x^{2}+y^{2}\right)^{2}}\right) .
\end{aligned}
$$

Using this in Eq. (B11) finally yields

$$
\begin{aligned}
{[h]_{n n^{\prime}, m}=} & -4 \pi \frac{d}{R} \frac{x_{1 n}}{J_{2}\left(x_{1 n}\right)} \frac{x_{1 n^{\prime}}}{J_{2}\left(x_{1 n^{\prime}}\right)} \frac{(2 m) !}{(m !)^{2} 2^{2 m}} \\
& \times \int_{0}^{1} x d x \int_{0}^{1} y d y J_{0}\left(x_{1 n} x\right) \\
& \times J_{0}\left(x_{1 n^{\prime}} y\right) \frac{(x y)^{m}}{\left(x^{2}+y^{2}\right)^{m+1 / 2} 2} F_{1} \\
& \times\left(\frac{2 m+1}{4}, \frac{2 m+3}{4}, m+1, \frac{4 x^{2} y^{2}}{\left(x^{2}+y^{2}\right)^{2}}\right) .
\end{aligned}
$$

This integral giving the matrix elements has to be evaluated numerically. The Coulomb-type singularity expected at $x=y$, as well as the oscillatory character of the integrand means that some care is needed while performing the integration. Here a VEGAS ${ }^{32}$-type adaptive Monte Carlo integrations algorithm was used. Notice that the set of functions in Eq. (B4) form a complete orthonormal basis set. If we calculate the operator matrix elements using the whole infinite set, we will get, in principle, the exact eigenvalues. A rough estimate, on the other hand, can be found by simply calculating the 
expectation value of the operator in the state $\psi_{n, m}: N_{n, m}$ $=-\left(\psi_{n, m}, \hat{h}_{r} \psi_{n, m}\right)$. This corresponds to the diagonal elements of the operator matrix. To increase the accuracy of the eigenvalues, a $15 \times 15$ matrix $[h]_{\widetilde{n} \tilde{n}^{\prime}, m}^{(15)}$ was computed and diago- nalized. We then find the higher-order approximation for the eigenvalues: $N_{n, m}^{(15)}=-\left\{\operatorname{spec}\left([h]_{\widetilde{n} \tilde{n}^{\prime}, m}^{(15)}\right)\right\}_{n}$, which does further improve the agreement with experiment as discussed in the text.
${ }^{1}$ Lord Rayleigh, The Theory of Sound, 2nd ed., revised and enlarged (Dover Publications, New York, 1945), Vol. 1, pp. 306394.

${ }^{2}$ T. D. Rossing, Am. J. Phys. 50, 271 (1982).

${ }^{3}$ J. B. Pendry and D. R. Smith, Am. J. Phys. 57(6), p. 37 (2004), and references therein.

${ }^{4}$ J. B. Pendry and D. R. Smith, Phys. Rev. Lett. 90, 029703 (2003).

${ }^{5}$ P. V. Parimi, W. T. Lu, P. Vodo, J. Sokoloff, J. S. Derov, and S. Sridhar, Phys. Rev. Lett. 92, 127401 (2004).

${ }^{6}$ Hideo Kosaka, Takayuki Kawashima, Akihisa Tomita, Masaya Notomi, Toshiaki Tamamura, Takashi Sato, and Shojiro Kawakami, Phys. Rev. B 58, R10 096 (1998).

${ }^{7}$ R. Y. Chiao, Phys. Rev. A 48, R34 (1993).

${ }^{8}$ R. Damon and J. Eshbach, J. Appl. Phys. 31, 104S (1960).

${ }^{9}$ P. C. Fletscher and C. Kittel, Phys. Rev. 120, 2004 (1960).

${ }^{10}$ M. Sparks, Solid State Commun. 8, 731 (1970).

${ }^{11}$ J. F. Dillon Jr., J. Appl. Phys. 31, 1605 (1960).

${ }^{12}$ G. Gubbiotti, G. Carlotti, T. Okuno, T. Shinjo, F. Nizzoli, and R. Zivieri, Phys. Rev. B 68, 184409 (2003).

${ }^{13}$ J. P. Park, P. Eames, D. M. Engebretson, J. Berezovsky, and P. A. Crowell, Phys. Rev. Lett. 89, 277201 (2002).

${ }^{14}$ C. Bayer, J. P. Park, H. Wang, M. Yan, C. E. Campbell, and P. A. Crowell, Phys. Rev. B 69, 134401 (2004).

${ }^{15}$ S. O. Demokritov, B. Hillebrands, and A. N. Slavin, Phys. Rep. 348, 441 (2001).

${ }^{16}$ G. N. Kakazei, P. E. Wigen, K. Yu. Guslienko, V. Novosad, A. N. Slavin, V. O. Golub, N. A. Lesnik, and Y. Otani, Appl. Phys. Lett. 85, 443 (2004).

${ }^{17}$ M. Buess, Y. Acremann, A. Kashuba, C. H. Back, and D. Pescia, J. Phys.: Condens. Matter 15, R1093 (2003).

${ }^{18} \mathrm{http}: / /$ llgmicro.home.mindspring.com/

${ }^{19}$ http://math.nist.gov/oommf/

${ }^{20}$ J. Raabe, R. Pulwey, R. Sattler, T. Schweinbck, J. Zweck, and D. Weiss, J. Appl. Phys. 88, 4437 (2000).
${ }^{21}$ Y. Acremann, C. H. Back, M. Buess, O. Portmann, A. Vaterlaus, D. Pescia, and H. Melchior, Science 290, 492 (2000).

${ }^{22}$ W. K. Hiebert, A. Stankiewicz, and M. R. Freeman, Phys. Rev. Lett. 79, 1134 (1997).

${ }^{23}$ S. Tamaru, J. A. Bain, R. J. M. van de Veerdonk, T. M. Crawford, M. Covington, and M. H. Kryder, J. Appl. Phys. 91, 8034 (2002).

${ }^{24}$ R. J. Hicken, A. Barman, V. V. Kruglyak, and S. Ladak, J. Phys. D 36, 2183 (2003).

${ }^{25}$ M. Buess, R. Höllinger, T. Haug, K. Perzlmaier, U. Krey, D. Pescia, M. R. Scheinfein, D. Weiss, and C. H. Back, Phys. Rev. Lett. 93, 077207 (2004).

${ }^{26}$ J. P. Park, P. Eames, D. M. Engebretson, J. Berezovsky, and P. A. Crowell, Phys. Rev. B 67, 020403(R) (2003).

${ }^{27}$ J. Jorzick, S. O. Demokritov, B. Hillebrands, M. Bailleul, C. Fermon, K. Y. Guslienko, A. N. Slavin, D. V. Berkov, and N. L. Gorn, Phys. Rev. Lett. 88, 047204 (2002).

${ }^{28}$ J. D. Jackson, Classical Electrodynamics, 3rd ed. (Wiley, New York, 1999), p. 115.

${ }^{29}$ S. O. Demokritov, A. A. Serga, A. Andre, V. E. Demidov, M. P. Kostylev, B. Hillebrands, and A. N. Slavin, Phys. Rev. Lett. 93, 047201 (2004); V. Novosad, M. Grimsditch, K. Yu. Guslienko, P. Vavassori, Y. Otani, and S. D. Bader, Phys. Rev. B 66, 052407 (2002).

${ }^{30}$ J. D. Jackson, Classical Electrodynamics, 3rd ed. (Wiley, New York, 1999), p. 118.

${ }^{31}$ G. N. Watson, A Treatise on the Theory of Bessel Functions, 2nd ed. (Cambridge University Press, Cambridge, 1995), Chap. 13.43 , p. 407. The equation has an erroneous extra factor $\sqrt{\pi}$ on the right side of the equality.

${ }^{32}$ G. P. Lepage, J. Comput. Phys. 27, 192 (1978); Cornell Report No. CLNS 80-447 (unpublished).

${ }^{33}$ For modes smaller than one micron and higher mode numbers, the stronger effect of exchange interaction lead to deviations between theory and micromagnetic simulation. 